\title{
A day in the half-life of a protein
}

Researchers describe a method called bleach-chase to quantitatively measure the half-lives of fluorescently tagged proteins in human cancer cells.

One of the ways that a cell regulates itself is by removing proteins that it no longer needs or that are detrimental to its health. This happens by two major mechanisms: degradation (such as via the proteasome) or dilution of the protein owing to cell growth. Rapidly dividing cells such as bacteria mainly rely on dilution to remove proteins, but terminally differentiated mammalian cells mostly use degradation. Because protein removal is often dominated by one process or another depending on the cell type, "the balance between degradation and dilution is often ignored," says Eran Eden. "We wanted a way to measure both."

Eden, a recently minted Ph.D. from Uri Alon's group at the Weizmann Institute of Science, Alon and their colleagues considered the traditional methods for measuring protein degradation rates, which include pulse-chase and translation inhibition. Although pulse-chase has been used for decades, this method requires radioactive labeling and protein-specific antibodies for detection. Protein synthesis inhibitors, in contrast, can greatly perturb the cell. The researchers wanted a non-radioactive method that would minimally perturb the cells and that could be scaled to follow the fate of a large number of proteins.

They came up with a method to measure protein half-lives that they call bleach-chase. They label the protein of interest with a fluorescent YFP tag and then shine light on the cells, just for long enough to bleach 10-60\% of the fluorophores; the short bleaching time does not substantially perturb the cells. "Conceptually, we can think of this as an 'anti-tag' that is generated at timepoint zero," explains Eden. "If you monitor the cells through time you will see that the new protein that is produced is the YFP-tagged protein." The problem with the anti-tag, however, is that it is invisible to fluorescence detection. Thus, to determine the protein removal rate, the researchers perform the experiment twice, once with bleaching and once without bleaching, using fluorescence time-lapse microscopy for detection. Then they simply subtract the fluorescence levels to obtain the dynamics of the 'invisible' proteins.
Because bleach-chase can be used to accurately quantify protein half-life, the method can help clearly identify which proteins are being actively degraded and which are being diluted as the cell divides. Eden, co-first author Naama Geva-Zatorsky, Alon and their colleagues have been working with human cancer cells, which have moderate growth rates, so the balance of degradation and dilution is important. In determining half-lives for 100 diverse proteins, they found that about $48 \%$ were regulated by degradation, $10 \%$ by dilution and the remaining $42 \%$ by a mixture of the two processes.

They then applied stress to the cells in the form of a chemotherapy drug, which slowed the growth rate, and looked at the effect this had on the protein removal rate. Whereas the degradation rate of shortlived proteins did not really change, the half-lives of the long-lived proteins, which are largely removed by dilution, became even longer. When they tested other stresses, which also slowed cell growth but at a different rate, they observed the same effect. "We think this is quite a general phenomenon," Eden says. "This is a simple principle that can help one understand a lot about the removal rate of a protein in response to different stresses." Notably, this result seems to suggest that cells do not use protein degradation to compensate for changes in the dilution rate.

The bleach-chase method should be useful to anyone who needs to measure protein half-lives. "Doing these experiments is very, very simple compared to pulse-chase," says Eden. Because the method allows one to generate results much more rapidly than with pulse-chase, "it can really open the ability to measure protein half-life on a large scale," he says. One drawback is that the proteins must be fluorescently tagged, which could alter the protein removal rate, but the researchers were careful to rule out this possibility with pulsechase control experiments.

As fluorescently tagged protein libraries are becoming available for more and more organisms, the method is likely to be widely applicable.

\section{Allison Doerr}

\section{RESEARCH PAPERS}

Eden, E. et al. Proteome half-life dynamics in living human cells. Science advance online publication (13 January 2011). 